\title{
Time domain modelling and stability analysis of complex thermoacoustic systems ${ }^{\dagger}$
}

\author{
M R Bothien*, J P Moeck, A Lacarelle, and C O Paschereit \\ Institute of Fluid Dynamics and Engineering Acoustics (Hermann-Föttinger-Institute), Technical University Berlin, \\ Berlin, Germany
}

The manuscript was received on 31 October 2006 and was accepted after revision for publication on 11 April 2007.

DOI: 10.1243/09576509JPE384

\begin{abstract}
A methodology allowing for a modular setup of complex acoustic systems is developed. The transfer behaviour of the individual subsystems is formulated in time domain. Subsystem descriptions can be obtained by analytical considerations, numerical methods, or experimental data. Once the complex subsystems have been characterized experimentally, changes in system geometry can be implemented easily by exchanging or adding subsystems. To validate the modelling approach, experiments are conducted in an acoustic test rig with a combustor-type geometry. Results are compared to predictions from the model, demonstrating accuracy in frequency and time domain. Application to thermoacoustic instabilities arising in lean-premixed combustion is given. The influence of a modified fuel distribution on an unstable operating point of a lean-premixed combustor is studied and validated with experimental data. Additionally, a study on the parameters governing the flame transfer function is performed to generate a stability map of a model combustor. An advantage of the state-space approach is that stability of a thermoacoustic system can be determined by simply solving a matrix eigenvalue problem. This is in strong contrast to the traditional approach, where the complete model is formulated in frequency domain with infinite-dimensional transfer functions. The time domain approach is based on the methodology presented by Schuermans et al. [1]. In contrast to their work, however, subsystems are not obtained from modal expansions but are characterized by using system identification techniques. Additionally, accuracy of the time domain model is verified by experiments.
\end{abstract}

Keywords: stability analysis, state-space system, thermoacoustics, time domain simulation

\section{INTRODUCTION}

As emission levels for modern gas turbines become more and more restrictive, the gas turbine industry stays abreast of changes by using lean-premixed combustion. However, the leaner a combustion system operates the more it is prone to suddenly occurring large pressure oscillations. These so-called thermoacoustic instabilities arise from the interaction of

\footnotetext{
${ }^{*}$ Corresponding author: Institute of Fluid Dynamics and Engineering Acoustics (Hermann-Föttinger-Institute), Technical University Berlin, Müller-Breslau-Straße 8, 10623 Berlin, Germany. email: mirko.bothien@tu-berlin.de

${ }^{\dagger}$ Extended version of a paper originally presented at the 2006 Conference on Modelling Fluid Flow (CMFF'06), Budapest University of Technology and Economics, 6-9 Sepetember 2006.
}

unsteady heat release and the acoustic field in the engine. If the two mechanisms constructively interfere, high amplitude pressure pulsations occur, which have a detrimental effect on the combustion process [2]. Among others, Dowling and Hubbard [3] expressed this fact in terms of an acoustic energy equation, which states that perturbations grow if their net energy gain from the combustion-acoustic interaction is greater than their losses across the system boundaries and due to dissipation.

Thermoacoustic instabilities are a major issue in gas turbines - both in stationary and aerospace applications but also in boilers and furnaces $[\mathbf{2}, \mathbf{4}, \mathbf{5}]$. Strong pressure oscillations cause higher emissions of pollutants $\left(\mathrm{NO}_{x}\right.$ and $\left.\mathrm{CO}\right)$, decrease the engine performance and significantly increase the noise level. If the fluctuations are high enough, they can even cause structural damage $[2,6,7]$. 
To predict unreliable and unsafe operation, it is necessary to describe how the system's acoustic field couples with the heat release. A model of the acoustic properties, supplemented by information on the flame response, allows for prediction and control of combustion instabilities. Thus, maintenance rates can be extended and the operating process can be improved. Furthermore, a model can also be used to formulate design constraints to new burner developments.

As full-scale engine tests are extremely costly and time-consuming, detailed investigations of thermoacoustic phenomena are commonly conducted in single burner test rigs. Due to the high complexity of geometry and interaction mechanisms in combustion systems, a comprehensive description of their acoustic field is cumbersome [1]. Although detailed numerical simulations (taking into account reaction, flow, and acoustics) were shown to be able to accurately calculate the thermoacoustic interaction mechanisms in single burner configurations [8], time expense, and computing power requirements are very high. Schuermans et al. [9] decreased the computational effort by coupling low-order acoustic models, representing complex impedance boundary conditions, to a computational fluid dynamics (CFD) solver. Especially if stability has to be assessed for a high number of parameter combinations (as shown in section 5), the use of a CFD code is not practicable. Moreover, as a CFD simulation is only able to observe the dominant mode of the instability, it is usually not possible to assess more linearly unstable modes [10]. Additionally, marginally stable modes cannot be detected, which is in contrast to the network approach described in the following. Sattelmayer and Polifke $[11,12]$ stated that it is extremely difficult to derive suitable measures to improve the system stability using results of large eddy simulation (LES) calculations, although these are able to give detailed information on the periodic reacting flow. Therefore, methods based on analytical modelling will remain the most appropriate stability assessment tool in the relatively near future.

One further step to reduce computational effort is to use low-order models to describe the whole combustion system. The common approach is to divide the total system into several subsystems, which are described by transfer functions accounting for plane wave propagation. Connection of these subsystems results in a low-order representation of the total system's acoustic properties. The network approach has the main advantages that information about the system's acoustics and insight into the physical processes can be obtained very quickly [13], which is of major importance in early design phases. Furthermore, changes in geometry or setup, as for example the performance of different burners, can easily be implemented in the model by simply exchanging a subsystem. Due to this modular setup, it is possible to incorporate an experimentally determined flame transfer function in the model. The flame-acoustic interaction is crucial for the stability of thermoacoustic systems and it is far from trivial to represent the linear flame response accurately in a CFD computation. A drawback of this approach is the limitation to plane wave acoustics. To overcome this problem, multi-dimensional models have to be considered. Evesque and Polifke [13] set up a two-dimensional low-order network, which was validated against a three-dimensional finite element method (FEM) solver showing good agreement.

Traditionally, stability analysis of thermoacoustic systems is accomplished by setting up a low-order network model, which is then investigated in the frequency domain. This is either done by solving the system's dispersion relation $[\mathbf{1 4}, \mathbf{1 5}]$, or by graphical methods (Nyquist plots) as described by Sattelmayer and Polifke [11, 12]. Both the graphical approach and finding the roots of the dispersion relation are generally not trivial but rather time-consuming tasks, especially if stability maps have to be generated [16].

In contrast to the traditional frequency domain approach, another procedure is followed here, by modelling the acoustic properties of the system in the time domain. Therefore, all subsystems are characterized in state-space form. For the resulting state-space network system, only a matrix eigenvalue problem has to be solved to perform a stability analysis, which is numerically straightforward. Schuermans et al. [7] also described the system in state-space form, however, they used modal expansion techniques to describe the individual subsystems.

Once a model for the thermoacoustic properties of the combustion system is at hand, control strategies can be implemented to suppress the feedback cycle. These strategies can either be passive by implementing changes in the combustor geometry or introduction of damping devices (e.g. Helmholtz or $\lambda / 4$-resonators) $[17,18]$, or active by means of openor closed-loop control [3, 19].

The remaining parts are structured as follows: sections 2 and 3 deal with the characterization of the subsystems in state-space form. The modelling approach is validated against experimental data in section 4 . Application to thermoacoustic stability analysis is then given in section 5 . Here, results from frequency domain and state-space calculations are also compared. In section 6 , a simulation model is used to predict the stabilizing influence of a modified fuel distribution in an experimental lean-premixed combustor. 


\section{STATE-SPACE REPRESENTATION OF ACOUSTIC SUBSYSTEMS}

As in the frequency domain transfer matrix approach [20], it is assumed that only plane waves propagate between two subsystems. This assumption is valid for elements that are coupled via ducts, for which the frequencies considered are below the cut-on frequency of the first non-planar mode. Neglecting entropy waves, this results in two degrees of freedom for all coupling planes. The variables accounting for that can be either acoustic pressure $p$ and velocity $v$ or the Riemann invariants of the plane wave field, denoted here with $f$ and $g$ for downstream and upstream travelling waves, respectively. The primitive variables are related to the Riemann invariants by $p=f+g$ and $v=f-g$. Here (as in the following), the acoustic pressure has been scaled with the characteristic impedance $\rho c$, the product of density and speed of sound.

Subsequently, acoustic systems are characterized using the scattering form, i.e. the $f$-wave upstream and the $g$-wave downstream are treated as inputs and, accordingly, the $f$-wave downstream and the $g$-wave upstream are obtained as outputs. The generic acoustic element in state-space form can then be written as

$$
\begin{aligned}
& \dot{\boldsymbol{x}}=\mathbf{A} \boldsymbol{x}+\mathbf{B}\left[\begin{array}{l}
f_{\mathrm{u}} \\
g_{\mathrm{d}}
\end{array}\right] \\
& {\left[\begin{array}{l}
f_{\mathrm{d}} \\
g_{\mathrm{u}}
\end{array}\right]=\mathbf{C} \boldsymbol{x}+\mathbf{D}\left[\begin{array}{l}
f_{\mathrm{u}} \\
g_{\mathrm{d}}
\end{array}\right]}
\end{aligned}
$$

where subscripts $\mathrm{u}$ and $\mathrm{d}$ denote upstream and downstream positions, respectively. A, B, C, and $\mathbf{D}$ are time-invariant $N \times N, N \times 2,2 \times N$, and $2 \times 2$ matrices, respectively, and $\boldsymbol{x}$ is the $N$-dimensional state vector. The dimension of the state depends on the acoustic subsystem to be characterized and has to be chosen sufficiently large so that the system's frequency response is represented with acceptable accuracy in the frequency range considered. An inlet or exit boundary condition can be written similar to equation (1) as a single input single output (SISO) system.

\section{SUBSYSTEM CHARACTERIZATION}

The modular setup allows for different ways of describing the subsystems, i.e. by analytical considerations, experimental data, or numerical methods (e.g. using FEM [21]). The subsystems' transfer functions relate the incoming and outgoing acoustic waves. Usually, acoustic subsystem characterization based on FEM computations or experiments results in frequency domain data. In the frequency domain the relation of the Riemann invariants is represented by the scattering matrix $\mathbf{S}$

$$
\left[\begin{array}{l}
f_{\mathrm{d}} \\
g_{\mathrm{u}}
\end{array}\right]=\left[\begin{array}{ll}
S_{11} & S_{12} \\
S_{21} & S_{22}
\end{array}\right]\left[\begin{array}{l}
f_{\mathrm{u}} \\
g_{\mathrm{d}}
\end{array}\right]
$$

where the elements $S_{i j}$ are complex-valued functions of frequency.

Once a subsystem's acoustic transfer function is given in the frequency-dependent form, equation (2), it can easily be transformed into the corresponding state-space form (equation (1)). The elements of the matrices of this state-space realization depend on how the system's state-space variables are defined. The same input-output behaviour can be obtained from different definitions of the system's state variables. If these variables are determined from the poles of a partial fraction expansion of the elements $S_{i j}$, which is mostly the case in this article, the system matrix $\mathbf{A}$ (equation (1)) assumes the so-called Jordan canonical form. The diagonal elements then comprise the poles of the partial fraction expansion. Detailed information on how this and other state-space formulations can be obtained from a transfer function in frequency domain can be found in reference [22]. In the following sections 4 and 5 , more information on the specific form of the transfer function of each individual subsystem is given. Here, either the scattering matrix, transfer function, or state-space form is chosen, depending on which formulation is more descriptive.

\subsection{Analytical description}

The description of elements with simple geometries (e.g. ducts) is straightforward. Neglecting sound absorption at the walls and in the fluid, a duct only represents a time-delay $\left(\mathrm{e}^{-\mathrm{i} \omega \tau}\right)$ in the system, i.e. an acoustic wave is transmitted with a delay, depending on the elements length and the speed of sound.

It is not possible to exactly describe a system with time-delay with a rational transfer function. Therefore, it has to be approximated, e.g. with a Padé approximation of the exponential function. The order of the Padé approximation, which is necessary for a sufficient accuracy, depends on the desired frequency range $(100 \mathrm{~Hz}-1000 \mathrm{~Hz}$ in the case considered here) and the time-delay. A duct with a length of $0.5 \mathrm{~m}$ at ambient conditions requires at least an order of 8 in the frequency band mentioned above.

\subsection{Experimental description}

Transfer functions of more complex subsystems can be determined by means of experiments. For this purpose, the element is regarded as a black box, for which the four unknown elements of the scattering matrix (equation (2)) have to be determined. This procedure 
has been applied previously [23-25] and thus only a short description is presented here.

The system given by equation (2) consists of two equations. Thus, two linearly independent states are necessary to solve for the four unknowns $S_{i j}$. The test states are created by successive acoustic excitation on both sides of the element. To obtain the Riemann invariants from sound pressure measurements at least two microphones at two different axial locations in a duct are necessary. In the presence of measurement uncertainty, better results are obtained by acquiring the pressure at more $(n)$ positions. Having data of more than two microphones, the problem is over-determined. This approach is called the multimicrophone method (MMM) [24]. The up- and downstream travelling waves are fitted to the measured quantities by means of the least squares method

$$
\left[\begin{array}{c}
f \\
g
\end{array}\right]=\mathbf{Z}^{+}\left[\begin{array}{c}
p_{1} \\
\vdots \\
p_{n}
\end{array}\right]
$$

where $\mathbf{Z}$ is given by

$$
\mathbf{Z}=\left[\begin{array}{cc}
\mathrm{e}^{-\mathrm{i} \omega \frac{x_{1}}{c+u_{0}}} & \mathrm{e}^{\mathrm{i} \omega \frac{x_{1}}{c-u_{0}}} \\
\vdots & \vdots \\
\mathrm{e}^{-\mathrm{i} \omega \frac{x_{n}}{c+u_{0}}} & \mathrm{e}^{\mathrm{i} \omega \frac{x_{n}}{c-u_{0}}}
\end{array}\right]
$$

Here, $x_{i}$ is the distance of microphone $i$ to the reference plane, i.e. the location where $f$ and $g$ are determined. $\mathbf{Z}^{+}$denotes the pseudo-inverse of $\mathbf{Z}$

$$
\mathbf{Z}^{+}=\left(\mathbf{Z}^{\mathrm{T}} \mathbf{Z}\right)^{-1} \mathbf{Z}^{\mathrm{T}}
$$

where the superscripts -1 and $\mathrm{T}$ denote the inverse and the conjugate transpose of a matrix, respectively.

\section{EXPERIMENTAL VALIDATION OF THE STATE-SPACE APPROACH}

\subsection{Experimental setup}

The network approach based on state-space models described in the preceding section was validated in an acoustic test rig. Figure 1 shows a schematic overview of the experimental facility.
The model for the test rig is split into subsystems, whose scattering matrices were determined experimentally (burner, up-, and downstream end) and analytically (ducts). Interconnection of the subsystems yields a model for the test rig, which can be used to predict its acoustic field.

The up- and downstream ends are equipped with circumferentially mounted loudspeakers, which allow for acoustic excitation of the rig. With these loudspeakers, it is possible to adjust the linearly independent test states that are necessary to experimentally obtain a system's scattering matrix. For the determination of the acoustic field, the test rig's duct sections are instrumented with $1 / 4^{\prime \prime}$ condenser microphones. There are three microphones upstream of the burner element and four downstream, thus allowing for the application of the MMM.

\subsection{Setup of the simulation model}

\subsubsection{Loudspeaker elements}

The speaker elements' transfer functions were determined experimentally. The elements have two inputs and one output. In the case of the upstream element, the $g$-wave and the loudspeaker voltage $u_{1}$ are the inputs while the $f$-wave represents the output. In the frequency domain this can be written as

$$
f=R g+G u_{1}
$$

where $R$ is the reflection coefficient and $G$ the transfer function of the loudspeaker's controlling input voltage to the outgoing $f$-wave. Equation (6) implies the assumption that the contribution of the reflection coefficient and the loudspeaker transfer function can be linearly superimposed. Results shown in section 4.3 confirm the validity of this assumption.

Similar to the approach described previously, two excitation states have to be generated. This is achieved using loudspeaker excitation at each side separately. Downstream excitation (index A) yields

$$
f_{\mathrm{A}}=R g_{\mathrm{A}}
$$

from equation (6) as $u_{1}$ is zero. This allows for direct calculation of the reflection coefficient $R$. Excitation

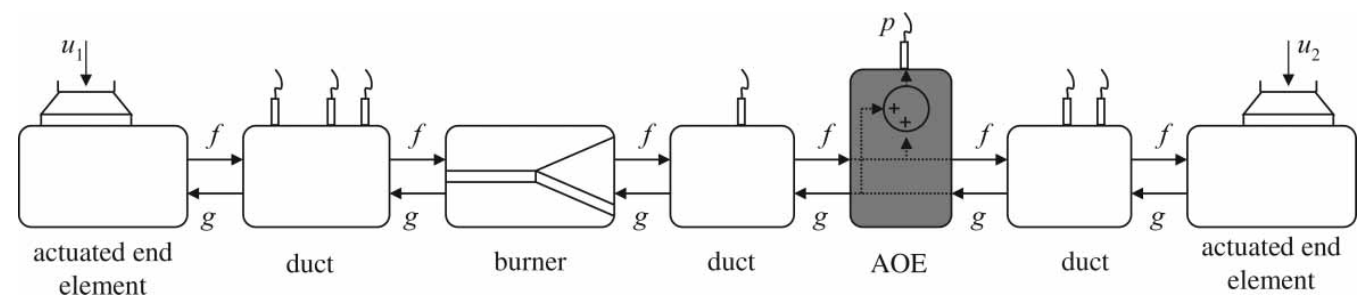

Fig. 1 Schematic overview of the acoustic test rig splitted up into its subsystems 


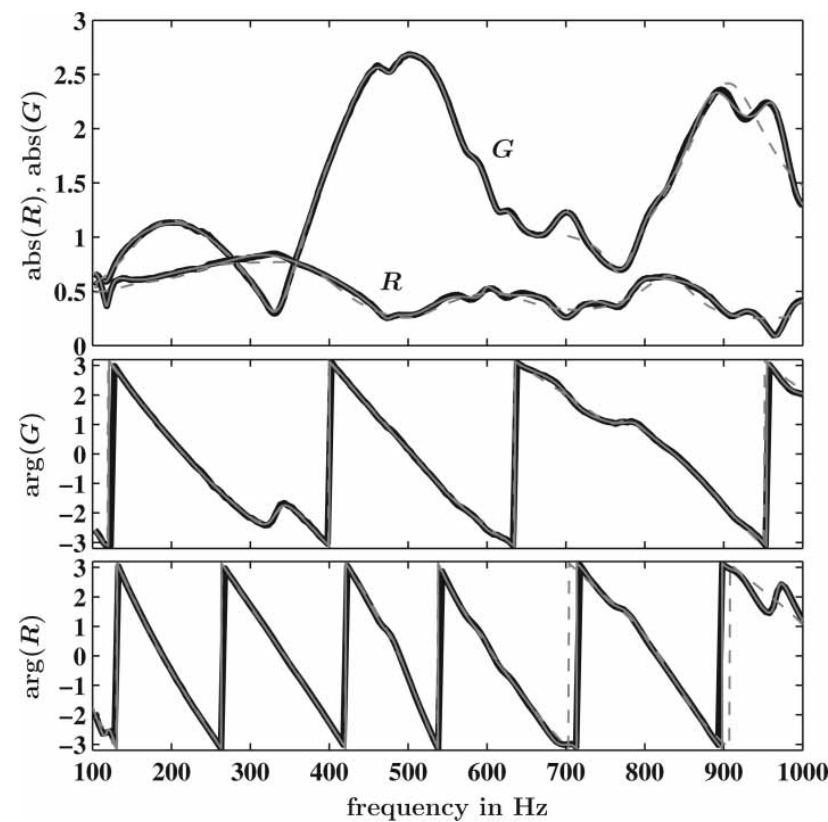

Fig. 2 Loudspeaker transfer function $(G)$ and reflection coefficient $(R)$ for measured values (black), model with 30 states (grey), and model with 15 states (grey dashed). Top: absolute value, middle: phase of $G$, bottom: phase of $R$

with the upstream loudspeaker (index B) and solving for $G$ gives

$$
G=\frac{f_{\mathrm{B}}-R g_{\mathrm{B}}}{u_{1}}
$$

where $R$ is calculated from equation (7).

With complex values for $R$ and $G$ calculated at discrete frequencies, frequency domain system identification algorithms [26, 27] were used to obtain state-space models. As stated before, the frequencydependent transfer functions are approximated by partial fraction expansions. The number of poles used for this expansion determines the number of states of the state-space system. Figure 2 depicts gain and phase of $R$ and $G$. Results for measured data as well as for models identified with 30 states and 15 states are shown.

As can be seen in Fig. 2, measured and computed curves for gain and phase of the reflection coefficient $R$ modelled with 30 states match almost perfectly. In the frequency regime above $700 \mathrm{~Hz}$, the model obtained with 15 states shows minor deviations. In the case of the loudspeaker's transfer function $G$, excellent results are obtained with 30 states. With 15 states, the results are fair except for some frequency bands, in which $G$ has several local extremal values. Regarding the phase, both models agree well with the measured values. The larger slope of $R$, i.e. the larger time-delay, is due to the position of the loudspeaker. The incident wave travels to the upstream element's end and back to the reference plane, whereas the emitted wave of the loudspeaker has to travel a much shorter distance.

Up to this point, only two SISO state-space systems exist. These have to be concatenated to a double input single output state-space system, which completely describes the upstream end

$$
\begin{aligned}
& \dot{\boldsymbol{x}}=\left[\begin{array}{cc}
\mathbf{A}_{R} & 0 \\
0 & \mathbf{A}_{G}
\end{array}\right] \boldsymbol{x}+\left[\begin{array}{cc}
\mathbf{B}_{R} & 0 \\
0 & \mathbf{B}_{G}
\end{array}\right]\left[\begin{array}{c}
g \\
u_{1}
\end{array}\right] \\
& f=\left[\begin{array}{ll}
\mathbf{C}_{R} & \mathbf{C}_{G}
\end{array}\right] \boldsymbol{x}+\left[\begin{array}{ll}
\mathbf{D}_{R} & \mathbf{D}_{G}
\end{array}\right]\left[\begin{array}{c}
g \\
u_{1}
\end{array}\right]
\end{aligned}
$$

Indices $R$ and $G$ denote the matrices of the reflection coefficient's and the loudspeaker's state-space systems, respectively.

\subsubsection{Artificial output element}

The grey highlighted element in Fig. 1, is not part of the real system but only an artificial component of the model system. Through this, it is possible to access the signal of any microphone by placing the artificial output element (AOE) at the position of the desired microphone. The element is modelled in state-space as a static gain without additional dynamics

$$
\left[\begin{array}{c}
f_{\mathrm{d}} \\
g_{\mathrm{u}} \\
p
\end{array}\right]=\left[\begin{array}{ll}
1 & 0 \\
0 & 1 \\
1 & 1
\end{array}\right]\left[\begin{array}{l}
f_{\mathrm{u}} \\
g_{\mathrm{d}}
\end{array}\right]
$$

Besides the $\mathbf{D}$ matrix, all other matrices of the statespace system (A, B, and C) are empty. The output element has a physical length of zero. The up- and downstream travelling waves are neither delayed nor changed in amplitude and therefore, the output element does not influence the acoustic behaviour of the total system.

\subsubsection{Interconnected complete system}

The outputs of each subsystem have to be connected with the inputs of its adjacent subsystems. Linking together the subsystems' state-space representations results in one single state-space model for the complete system. The interconnection is exemplarily shown for two adjacent subsystems of the acoustic test rig.

The transfer behaviour of a subsystem defines the relation between incident and outgoing waves. By connecting the two subsystems 'duct' and 'burner' (Fig. 3), one system is obtained with the Riemann invariants $f_{1}$ and $g_{3}$ as inputs and $g_{1}$ and $f_{3}$ as outputs. Thus, the two systems of equations describing the individual subsystems are now combined to one, representing the transfer behaviour of the connected system. This combination is a matrix operation referred to as the 


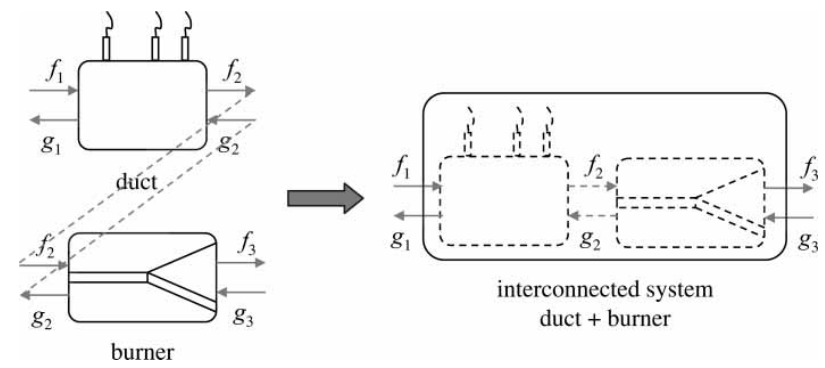

Fig. 3 Interconnection of two subsystems by means of the Redheffer Star Product

Redheffer Star Product [7, 22]. Applied to all subsystems of Fig. 1, this method yields one single state-space expression of the total system. Interconnection of the individual state-space systems can conveniently be done with built-in MATLAB routines.

Interconnecting all subsystems resulted in a system with an order of 200 . To reduce the order of the complete system, model reduction techniques were used [28], allowing the elimination of all states with negligible influence on the input-output behaviour. Through this, the model's order was reduced to 60 states exhibiting only a slight deviation in frequency response compared to the higher order system.

\subsection{Comparison of model and experiments}

Top and middle frames in Fig. 4 depict the transfer function of the input voltage of the upstream loudspeaker $u_{1}$ to the second microphone downstream of the burner for the reduced order system. This is the position, where the AOE is located in the simulation model.

Note that the grey curves in Fig. 4 show gain and phase of the transfer function obtained from the interconnection of the subsystems' state-space representations. The individual state-space formulations of the seven subsystems (up- and downstream end, burner element, ducts, and output element) were linked to one state-space system.

Only for frequencies below $150 \mathrm{~Hz}$, around $470 \mathrm{~Hz}$, and above $900 \mathrm{~Hz}$, a slight deviation in magnitude is observed. Regarding the phase, the results are even better, showing an observable deviation only at $300 \mathrm{~Hz}$. It is important to note here, that the modelled frequency response shown in Fig. 4 was not simply identified from the experimental data but was predicted based on the individual subsystem models.

The interconnected state-space model of the complete system - reduced to 60 states - was used to simulate the pressure fluctuations at the defined microphone position. For this purpose, the upstream loudspeaker was driven with a signal, which consisted of three sines (all the same in amplitude) with arbitrary frequencies set to 203,440 , and $580 \mathrm{~Hz}$. White
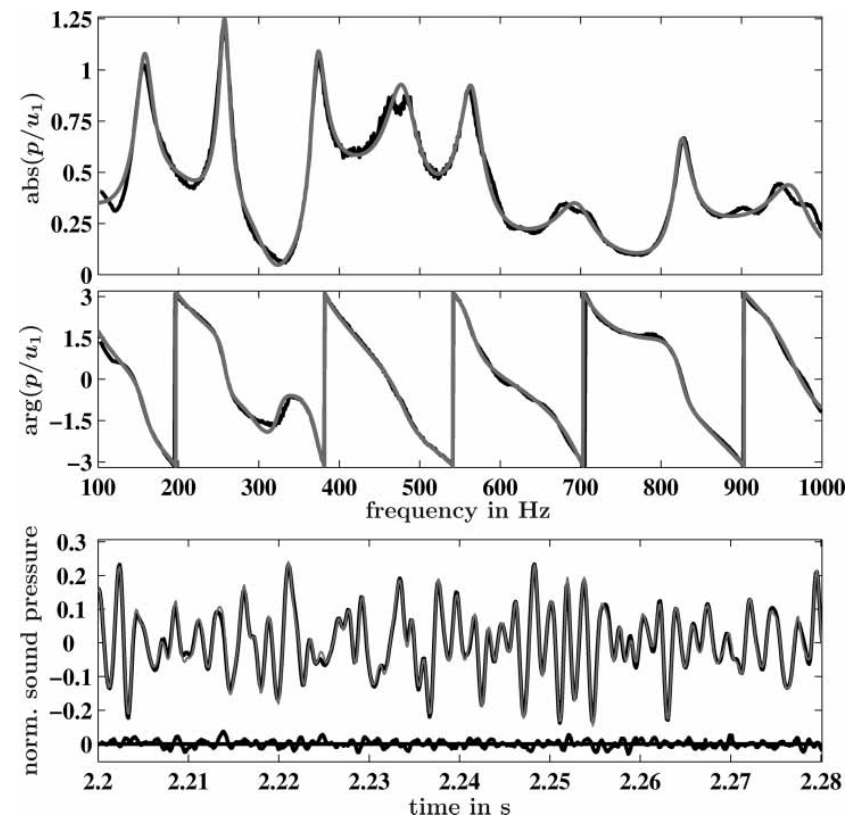

Fig. 4 Transfer function of input voltage $u_{1}$ (upstream loudspeaker) to sound pressure at position of output element; measured (black), interconnected model (grey). Top: magnitude, middle: phase, bottom: normalized sound pressure amplitude for measured (black) and predicted (grey) data and their deviation

noise, band-pass filtered between 100 and $600 \mathrm{~Hz}$, superimposed these sines.

Time responses of measured and predicted pressure fluctuations are also shown in Fig. 4 (bottom). Both signals show nearly perfect correspondence in phase and in amplitude. For clarity, their difference is also plotted (same scale). A comparison of measured and predicted data obtained with downstream excitation or excitation on both sides exhibited equally good results.

\section{APPLICATION TO THERMOACOUSTIC STABILITY ANALYSIS}

\subsection{Stability of thermoacoustic systems}

To assess the linear stability of a thermoacoustic system, the most commonly used procedure is the following [1, 11, 12].

1. The system is divided into different elements (duct, burner, etc.).

2. Transfer matrices for the individual elements are set up in frequency domain.

3. All transfer matrices are assembled in a system matrix accounting for proper matching conditions.

4. The system's dispersion relation det(system matrix) $=0$ is solved to obtain the system eigenvalues. 
5. Eigenvalues with negative imaginary parts indicate instability (time dependence $\sim \mathrm{e}^{\mathrm{i} \omega t}$ ), the corresponding real parts the frequency of the growing oscillation.

Concerning this procedure, some remarks should be made. In step 2, the transfer matrices are generally of a non-rational form, since the acoustic wave propagation is governed by a partial differential equation. Also, solving det (system matrix) $=0$ will only be possible, if all transfer matrices are available in analytical form. Except for academic examples this will not be the case. At least some elements will have to be determined from CFD or FEM computations or experimentally. However, even if closed-form expressions would be available for all transfer matrices involved, solving for the system's eigenvalues can be quite cumbersome. The dispersion relation is in general a complex-valued transcendental equation, rapidly increasing in length with network size.

The stability analysis based on a state-space description follows a procedure similar to the one given above for the traditional frequency domain approach. Connecting all subsystems results in a state-space system that represents the dynamics of the complete system. To assess stability, the temporal evolution of free oscillations has to be considered, which is governed by the dynamics matrix A. Similar to the roots of the transcendental dispersion relation in the traditional approach, the eigenvalues of the A matrix, $s$ (say), govern the stability of the system. $\operatorname{Re}(s)>0$ indicates an unstable mode with frequency $|\operatorname{Im}(s)| / 2 \pi$. This procedure was proposed by Schuermans et al. [1] .

From a numerical point of view, determining system stability from the state-space approach is straightforward, since calculating the eigenvalues from a given $\mathbf{A}$ matrix can be performed with standard linear algebra routines. In contrast to that, solving the transcendental dispersion relations resulting from frequency domain representations of thermoacoustic systems is usually not a trivial task, even for networks of medium complexity. These dispersion relations generally have an infinite number of solutions with only those in the low-frequency regime being of physical relevance. Evidently, the state-space approach can only provide a finite number of eigenvalues equal to the dimension of $\mathbf{A}$. This is due to the fact that the infinite-dimensional system has been reduced to a finite-dimensional state-space. In practice, however, the analysis will be limited to the low-frequency regime. One reason for this is that thermoacoustic instabilities most commonly appear in the form of low-frequency oscillations. In addition to that, the network approach based on plane wave matching is only valid for frequencies below the cut-on frequency of the first non-planar mode.

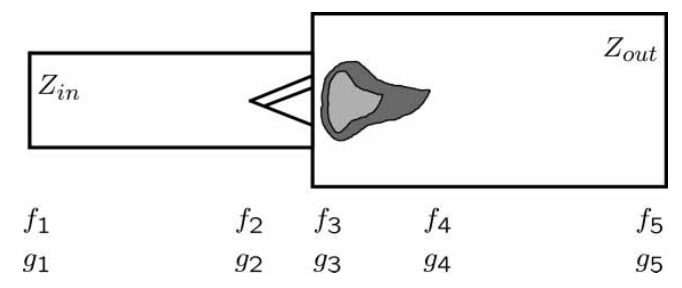

Fig. 5 Model system for stability analysis

\subsection{A thermoacoustic model system}

To assess feasibility of the state-space approach with regard to linear stability analysis, the stability of a thermoacoustic model system is investigated. In order to consider the accuracy of the state-space approach, the results have to be compared to a corresponding frequency domain calculation and therefore, only elements with fully analytical description are chosen.

The model system is shown in Fig. 5 and comprises the following subsystems:

(a) constant inlet impedance;

(b) uniform duct;

(c) $L-\zeta$ model for burner and area expansion;

(d) distributed time-lag model for the flame with variable mean time-delay and variable variance;

(e) uniform duct;

(f) Levine-Schwinger outlet impedance.

For the inlet impedance, a value of $Z_{\text {in }}=9$ is chosen, corresponding to a partially reflecting boundary with a reflection coefficient of 0.8 . The duct connecting the inlet and the burner has a length of $L_{\mathrm{c}}$, a temperature of $T_{c}$, and carries a mean flow with velocity $U$. All parameter values describing the model system can be found in Table 1.

The burner is represented by the $L-\zeta$ model (cf. [23]) for a compact element with the transfer matrix

$$
\mathbf{T}=\left[\begin{array}{cc}
1 & -\mathrm{i} k L_{\text {eff }}-\zeta M \\
0 & \alpha
\end{array}\right]
$$

$L_{\text {eff }}$ is the effective length of burner and area expansion, $\zeta$ the loss coefficient, and $k$ denotes the wave number of the plane wave field. The change in crosssectional area is given by $\alpha$. It can be noted here, that the upper right transfer function in equation (11) is improper [22] and, therefore, cannot be given in statespace form. However, the scattering form of the $L-\zeta$ model has a fully proper representation.

For the flame, a time-lag model with a Gaussian distribution of time-delays [29] was chosen. In this case, the transfer function coupling the velocities across the flame reads

$$
T_{22}=1+\left(\frac{T_{\mathrm{h}}}{T_{\mathrm{c}}}-1\right) \mathrm{e}^{-\mathrm{i} \omega \tau} \mathrm{e}^{-1 / 2 \sigma^{2} \omega^{2}}
$$


Table 1 Parameter values describing the model system

\begin{tabular}{llllllllll}
\hline Parameter & $L_{\mathrm{c}}$ & $T_{\mathrm{c}}$ & $U$ & $L_{\text {eff }}$ & $\zeta$ & $\alpha$ & $L_{\mathrm{h}}$ & $T_{\mathrm{h}}$ & $r$ \\
\hline Value & $0.95 \mathrm{~m}$ & $523 \mathrm{~K}$ & $10 \mathrm{~m} / \mathrm{s}$ & $0.13 \mathrm{~m}$ & 5 & 0.3025 & $1.3 \mathrm{~m}$ & $1573 \mathrm{~K}$ & $0.1 \mathrm{~m}$ \\
\hline
\end{tabular}

where $\tau$ and $\sigma$ denote mean and variance of the timedelay distribution.

The duct coupling the flame with the outlet boundary condition is assigned a length of $L_{\mathrm{h}}$ and a temperature of $T_{\mathrm{h}}$. The outlet is modelled as a long wave Levine-Schwinger exit condition [30], the (specific) impedance being given by

$$
Z_{\text {out }}=\frac{1}{4}(k r)^{2}+\mathrm{i} 0.6133 k r
$$

where $r$ is the outlet duct radius. The reflection coefficient is related to the impedance through the bilinear transform $R=(Z-1) /(Z+1)$. Thus, the condition relating $f$ and $g$ at the boundary is a proper transfer function and can be converted to a state-space realization. The ducts are assumed to support undamped acoustic wave propagation. As stated in section 3.1, a state-space model of the ducts is determined using Padé approximations.

To obtain stability characteristics, the procedure outlined in section 5.1 is followed. The roots of the dispersion relation are determined with an iterative solver working on a uniformly distributed random field of initial guesses in the complex $\omega$-plane. The search was restricted to a rectangular domain with $|\operatorname{Re}(\omega)| / 2 \pi<1000 \mathrm{~Hz}$ and $|\operatorname{Im}(\omega)|<100 \mathrm{rad} / \mathrm{s}$. As mentioned before, practical relevance justifies the restriction to the low-frequency regime. Also, highly damped modes (those with large $\operatorname{Im}(\omega)$ ) are not of interest and highly unstable ones are usually considered unphysical.

Figure 6 shows the complex eigenfrequencies of the model system in the specified interval for two sets of flame model parameters ( $\sigma$ variable, $\tau=7 \mathrm{~ms}$ ),

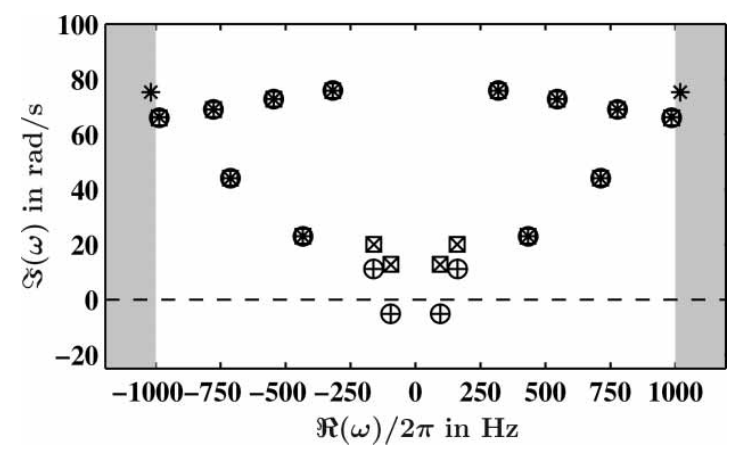

Fig. 6 Eigenvalues from frequency domain and state-space computation; $\circ, \square$ : frequency domain $\sigma_{\circ}=2 \mathrm{~ms}, \sigma_{\square}=2.5 \mathrm{~ms},+$, $\times$ : state-space $\sigma_{+}=2 \mathrm{~ms}, \sigma_{\times}=2.5 \mathrm{~ms}$ computed from frequency domain and state-space approach (see figure caption). It can be clearly seen that the frequency domain and the state-space calculations give identical results for both parameter combinations.

Furthermore, it can be observed how a slight increase in the variance of the time-delay distribution can lead to a stabilization of the system. For $\sigma=2 \mathrm{~ms}$ a low-frequency mode close to $100 \mathrm{~Hz}$ has a negative damping rate and is therefore linearly unstable. For a 25 per cent higher variance, the unstable mode and the least stable mode are shifted to higher damping rates resulting in a stable system $(\square, \times)$. Note also, how only the lowest frequency modes are affected by a change in the time-delay variance.

Having shown that the stability analysis based on the state-space model accurately reproduces the results from the traditional frequency domain approach, this method can be used to investigate the influence of the flame model parameters in more detail.

Figure 7 illustrates the dependence of system stability on the flame model parameters as obtained from the state-space description. Note that the stability problem has been solved 15000 times in $(\sigma, \tau)$ parameter space in less than an hour. This would be impossible to accomplish by using graphical methods based on Bode or Nyquist diagrams. Generating a stability map based on solutions of the frequency domain dispersion relation would be much more time-consuming as well. The greyscale coding indicates the growth rate of the least stable mode. The thick black line in the plot denotes the stability border. As can be seen, the highest growth rate can be found for a fully concentrated $(\sigma=0)$ time-delay of about

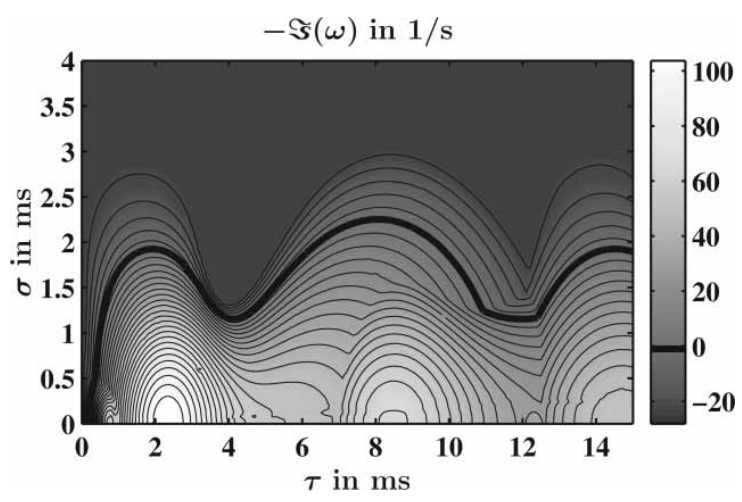

Fig. 7 Growth rate in 1/s of the least stable mode as a function of time-delay mean $\tau$ and variance $\sigma$ (equation (12)) 


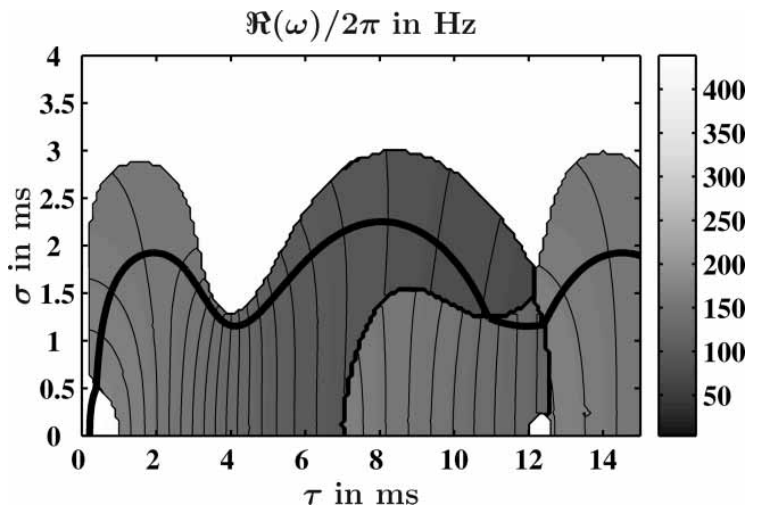

Fig. 8 Frequency in $\mathrm{Hz}$ of the least stable mode as a function of time-delay mean $\tau$ and variance $\sigma$ (equation (12))

$2.5 \mathrm{~ms}$. For this case, two more local maxima in growth rate can be identified at $\tau=8.5 \mathrm{~ms}$ and $\tau=14.5 \mathrm{~ms}$. An increase in time-delay variance has a purely stabilizing effect. For a fixed mean time-delay, the growth rate of the most unstable mode decreases monotonically with variance. In fact, for $\sigma>2.25 \mathrm{~ms}$ all modes are stable. The stabilizing effect of the time-delay variance is in accordance with Polifke et al. [31].

A contour plot of the frequency of the least stable mode as a function of time-delay mean and variance is shown in Fig. 8. The stability border is added again as a thick black line. In the unstable region, low-frequency modes in the range of $75-175 \mathrm{~Hz}$ are dominant. For certain values of $\tau$ and $\sigma$ isocontours of the dominant mode's frequency meet, indicating that there are at least two unstable eigenvalues. Note that lines of constant mode frequency have an essentially vertical trend. This corresponds to the fact that the frequency of the least stable mode does not change considerably with time-delay variance [31].

With respect to computing time, the stability analysis based on state-space models was recognized to be clearly more efficient than the frequency domain approach. Additionally, using the latter for parametric studies is not straightforward, since for all sets of parameters all roots of the dispersion relation in the domain considered must be located. This can only be ensured if a high number of initial guesses for the iterative solver is chosen. However, this results in a considerably higher computing time. Although graphical methods are a useful tool to investigate thermoacoustic stability for fixed parameter sets, they are not suitable for generating stability maps.

\section{APPLICATION TO AN ATMOSPHERIC COMBUSTOR TEST RIG}

In this section, the state-space methodology is applied to investigate the stability of an atmospheric

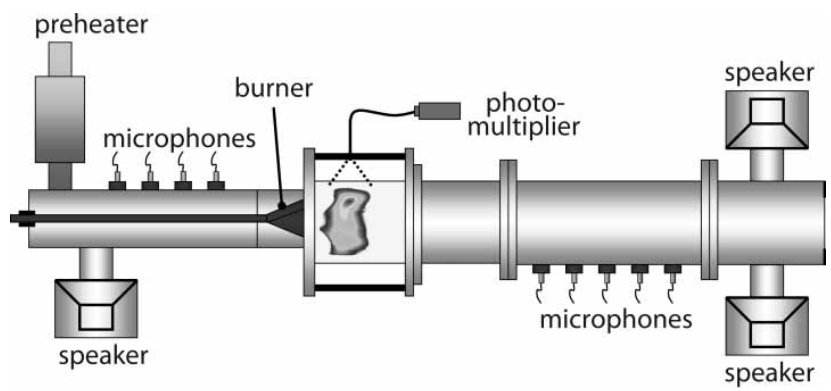

Fig. 9 Setup of combustion test rig with instrumentation

combustor test rig with a swirl stabilized burner. A schematic of the test rig is shown in Fig. 9.

The lean-premixed burner features aerodynamic flame stabilization through a vortex breakdown induced by strong swirl. Pressure field and $\mathrm{OH}^{*}$ chemiluminescence, an indicator of heat release [32], can be monitored. To investigate its thermoacoustic properties the test rig is equipped with loudspeakers and microphones mounted up- and downstream of the flame. A more detailed description of the test rig can be found in references [33] and [34]. In order to generate thermoacoustic instabilities, a resonance tube was attached to the combustion chamber. This resulted in a high amplitude quarter wave mode instability at $\sim 100 \mathrm{~Hz}$ for certain preheated, lean-premixed operating conditions.

Stability analysis based on the state-space approach was applied to investigate the impact of a fuel distribution modification on thermoacoustic system stability. The fuel distribution was modified as such, that the majority of the fuel (natural gas) was directed towards the outer flame region. This resulted in an enriched outer combustion zone, whereas the inner region was made leaner. For more details see reference [35].

The impact of the fuel distribution modification on the flame response was taken into account by means of the flame transfer function. Flame responses for both, the baseline and the modified case were measured at the operating conditions where stability was investigated. For the modified distribution, the phase response was quite similar to the standard case, however, in the frequency range of $80-150 \mathrm{~Hz}$, the amplitude response was considerably lower $[\mathbf{1 6}, \mathbf{3 5}]$.

In addition to the experimentally determined flame responses, a parametric model for the upstream reflection coefficient was identified and included in the model. This was considered necessary since the upstream acoustic boundary condition was rather complex due to the preheater and air supply geometry. The complete model was composed of 11 elements and had a state dimension of 120 .

System eigenfrequencies for the baseline and the modified case are shown in Fig. 10. The baseline 


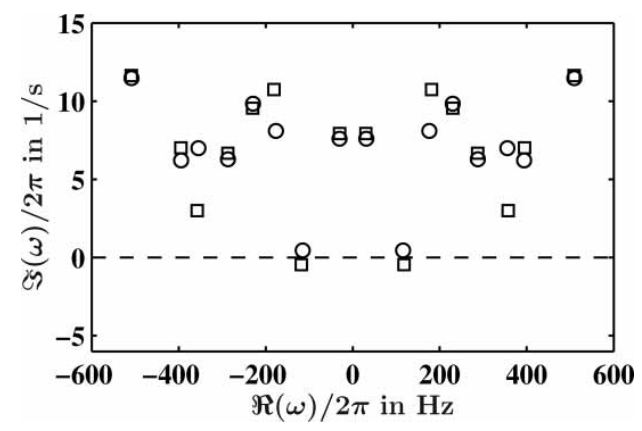

Fig. 10 Complex eigenfrequencies of system model; $\square$ : baseline, o: modified

case has an eigenvalue with negative imaginary part close to $100 \mathrm{~Hz}$, indicating an unstable mode. For the enriched outer combustion zone, the initially unstable eigenvalue, representing the quarter wave mode, moves across the real axis to the upper half plane. This implies that the system is now linearly stable.

The stabilizing effect of the enriched outer combustion zone could be verified in the experiment. Frequency spectra of the unsteady combustor pressure for the standard and the modified case are shown in Fig. 11.

The spectrum for the standard case exhibits a dominant peak at $100 \mathrm{~Hz}$ corresponding to the unstable quarter wave mode. In contrast to that, the spectrum for the modified case shows a significant reduction of the spectral peak amplitude (more than $20 \mathrm{~dB}$ ). It is difficult to judge if the system is actually stable since the pressure amplitudes at the quarter wave frequency were still high. Despite that, high pulsation amplitudes can also occur in a stable lightly damped system, which is driven by noise [36]. Compared to the standard case, the stabilizing effect of the modified fuel distribution, as predicted by the model, can be clearly seen in the experimental data. It should be also mentioned that enriching the outer combustion zone did not have a stabilizing effect for all operating conditions that were tested [35].

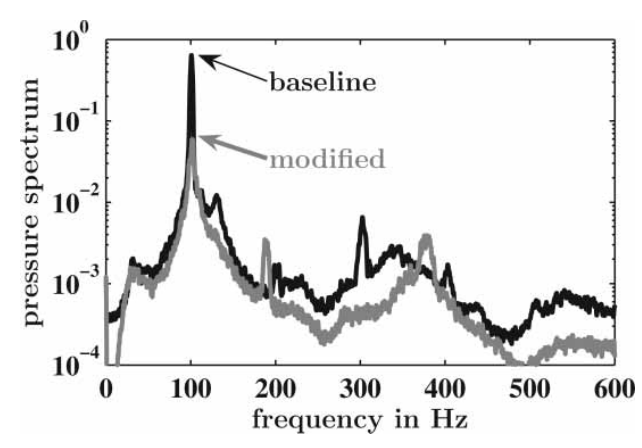

Fig. 11 Measured amplitude spectra of the unsteady combustor pressure for the baseline and the modified case

\section{SUMMARY}

A modular approach for modelling complex thermoacoustic systems based on state-space models was presented. Frequency and time domain results for a purely acoustic model system were compared to experimental data and proved the method to be of high accuracy.

Additionally, application to stability analysis of thermoacoustic systems was investigated. Results were compared to a standard frequency domain analysis of the same system. Exactly the same results were obtained with a fraction of computational effort compared to that of the standard procedure.

The impact of a modified fuel distribution on thermoacoustic stability of an experimental combustor was investigated using the state-space methodology. Based on the model, a stabilizing effect was predicted, which was confirmed by experimental data.

\section{REFERENCES}

1 Schuermans, B., Bellucci, V., and Paschereit, C. O. Thermoacoustic modeling and control of multi burner combustion systems. In Proceedings of the ASME Turbo Expo, Atlanta, USA, 2003, paper 2003-GT-38688.

2 Lieuwen, T. C. and Yang, V. (Eds) Combustion instabilities in gas turbine engines. In Progress in astronautics and aeronautics, vol. 210, 2005 (AIAA, Inc., Reston).

3 Dowling, A. P. and Hubbard, S. Instability in lean premixed combustors. Proc. Instn Mech. Engrs, Part A: J. Power and Energy, 2000, 214, 317-332.

4 Dowling, A. P. and Morgans, A. S. Feedback control of combustion oscillations. Annu. Rev. Fluid Mech., 2005, 37, 151-182.

5 Seume, J. R., Vortmeyer, N., Krause, W., Hermann, J., Hantschk, C.-C., Zangl, P., Gleis, S., Vortmeyer, D., and Orthmann, A. Application of active combustion instability control to a heavy duty gas turbine. Trans. ASME, J. Eng. Gas Turbines Power, 1998, 19(6), 549-566.

6 Sewell, J., Sobieski, P., and Beers, C. Application of continuous combustion dynamics monitoring on large industrial gas turbines. In Proceedings of the ASME Turbo Expo, Vienna, Austria, 2004, paper GT2004-54310.

7 Rea, S., James, S., Goy, C., and Colechin, M. J. F. On-line combustion monitoring on dry low $\mathrm{NO}_{x}$ industrial gas turbines. Meas. Sci. Technol., 2003, 14, 1123-1130.

8 Roux, S., Lartigue, G., Poinsot, T., Meier, U., and Bérat, C. Studies of mean and unsteady flow in a swirled combustor using experiments, acoustic analysis, and large eddy simulations. Combust. Flame, 2005, 141, 40-54.

9 Schuermans, B., Luebcke, H., Bajusz, D., and Flohr, P. Thermoacoustic analysis of gas turbine combustion systems using unsteady CFD. In Proceedings of the ASME Turbo Expo, Reno-Tahoe, USA, 2005, paper GT200568393. 
10 Polifke, W. Combustion instabilities. In Advances in aeroacoustics and applications, lecture series, VKI LS 2004-05, 2004 (Von Karman Institute, Brussels, Belgium).

11 Sattelmayer, T. and Polifke, W. Assessment of methods for the computation of the linear stability of combustors. Combust. Sci. Technol., 2003, 175, 453-476.

12 Sattelmayer, T. and Polifke, W. A novel method for the computation of the linear stability of combustors. Combust. Sci. Technol., 2003, 175, 477-497.

13 Evesque, S. and Polifke, W. Low-order modelling for annular combustors: validation and inclusion of modal coupling. In Proceedings of the ASME Turbo Expo, Amsterdam, The Netherlands, 2002, paper GT-2002-30064.

14 Stow, S. R. and Dowling, A. P. Thermoacoustic oscillations in an annular combustor. In Proceedings of the ASME Turbo Expo, New Orleans, USA, 2001, paper 2001-GT-0037.

15 Schuermans, B. B. H., Paschereit, C. O., Polifke, W., and van der Linden, J. H. Prediction of acoustic pressure spectra in combustion systems using swirl stabilized gas turbine burners. In Proceedings of the IGTI Turbo Expo, Munich, Germany, 2000, paper 2000-GT-0105.

16 Paschereit, C. O., Moeck, J. P., and Bothien, M. R. Statespace modelling of thermoacoustic systems for stability analysis and time domain simulation. In Proceedings of the 13th International Congress on Sound and Vibration, Vienna, Austria, 2006.

17 Dupère, I. D. J. and Dowling, A. P. The use of Helmholtz resonators in a practical combustor. Trans. ASME, J. Eng. Gas Turbines Power, 2005, 127, 268-275.

18 Paschereit, C. O. and Gutmark, E. The effectiveness of passive combustion control methods. In Proceedings of the ASME Turbo Expo, Vienna, Austria, 2004, paper GT-2004-53587.

19 McManus, K. R., Poinsot, T., and Candel, S. M. A review of active control of combustion instabilities. Prog. Energy Combust. Sci., 1993, 19, 1-29.

20 Munjal, M. L. Acoustics of ducts and mufflers, 1976 (Wiley \& Sons, Inc., New York).

21 Peat, K. S. Evaluation of four-pole parameters for ducts with flow by the finite element method. J. Sound Vib., 1982, 84(3), 389-395.

22 Zhou, K. and Doyle, J. Essentials of robust control, 1997 (Prentice Hall, Upper Saddle River, NJ).

23 Schuermans, B. B. H., Polifke, W., and Paschereit, C. O. Modeling transfer matrices of premixed flames and comparison with experimental results. In Proceedings of the ASME Turbo Expo, Indianapolis, USA, 1999, paper 1999-GT-0132.

24 Paschereit, C. O., Schuermans, B., Polifke, W., and Mattson, O. Measurement of transfer matrices and source terms of premixed flames. Trans. ASME, J. Eng. Gas Turbines Power, 2002, 124, 239-247.

25 Åbom, M. A note on the experimental determination of acoustical two-port matrices. J. Sound Vib., 1992, 155(1), 185-188.

26 Gustavsen, B. and Semlyen, A. Rational approximation of frequency domain responses by vector fitting. IEEE Trans. Power Deliv., 1999, 14(3), 1052-1061.

27 Kollár, I., Pintelon, R., and Schoukens, J. Frequency domain system identification toolbox for matlab: improvements and new possibilities. In Proceedings of the XI. IFAC/IFORS International Symposium on System Identification and Parameter Estimation, Fukuoka, Japan, 1997, pp. 991-994.

28 Antoulas, A. C. and Sorensen, D. C. Approximation of large-scale dynamical systems: an overview. Int. J. Appl. Math. Comput. Sci., 2001, 11(5), 1093-1121.

29 Schuermans, B., Bellucci, V., Guethe, F., Meili, F., Flohr, P., and Paschereit, C. O. A detailed analysis of thermoacoustic interaction mechanisms in a turbulent premixed flame. In Proceedings of the ASME Turbo Expo, Vienna, Austria, 2004, paper GT 2004-53831.

30 Levine, H. and Schwinger, J. On the radiation of sound from an unflanged circular pipe. Phys. Rev., 1948, 73(4), 383-406.

31 Polifke, W., Kopitz, J., and Serbanovic, A. Impact of the fuel time lag distribution in elliptical premix nozzles on combustion stability. In Proceedings of the 7th AIAA/CEAS Aeroacoustics Conference, Maastricht, The Netherlands, 2001, paper 2001-2104.

32 Haber, L. C., Vandsburger, U., Saunders, W. R., and Khanna, V. K. An examination of the relationship between chemiluminescent light emission and heat release rate under non-adiabatic conditions. In Proceedings of the ASME Turbo Expo, Munich, Germany, 2000, paper 2000-GT-0121.

33 Albrecht, P., Bauermeister, F., Bothien, M. R., Lacarelle, A., Moeck, J. P., Paschereit, C. O., and Gutmark, E. Characterization and control of lean blowout using periodically generated flame balls. In Proceedings of the ASME Turbo Expo, Barcelona, Spain, 2006, paper GT2006-90340.

34 Moeck, J. P., Bothien, M. R., Guyot, D., and Paschereit, C. O. Phase-shift control of combustion instability using (combined) secondary fuel injection and acoustic forcing. In Proceedings of the 1st Active Flow Control Conference, Berlin, Germany, 2006.

35 Lacarelle, A., Moeck, J., Konle, H., Nayeri, C. N., and Paschereit, C. O. Effect of the fuel/air mixing on NOx emissions and stability in a gas premixed combustion system. In Proceedings of the 45th AIAA Aerospace Science Meeting, Reno-Tahoe, USA, 2007, paper 2007-1417.

36 Banaszuk, A., Jacobson, C. A., Khibnik, A. I., and Mehta P. G. Linear and nonlinear analysis of controlled combustion processes. Part I: linear analysis. In Proceedings of the IEEE International Conference on Control Applications, Hawaii, USA, 1999, pp. 199-205.

\section{APPENDIX}

$\begin{array}{ll}\text { Notation } & \\ \text { A, B, C, D } & \text { state-space matrices }(-) \\ c & \text { speed of sound }(\mathrm{m} / \mathrm{s}) \\ f & \text { downstream travelling wave }(\mathrm{m} / \mathrm{s}) \\ g & \text { upstream travelling wave }(\mathrm{m} / \mathrm{s}) \\ k & \text { wave number }(1 / \mathrm{m}) \\ M & \text { Mach number }(-) \\ p & \text { acoustic pressure scaled with } \rho c(\mathrm{~m} / \mathrm{s}) \\ r & \text { radius }(\mathrm{m}) \\ R & \text { reflection coefficient }(-)\end{array}$




$\begin{array}{ll}s & \text { eigenvalue }(-) \\ \mathbf{S} & \text { scattering matrix }(-) \\ t & \text { time (s) } \\ \mathbf{T} & \text { transfer matrix }(-) \\ T & \text { temperature }(\mathrm{K}) \\ u & \text { loudspeaker voltage }(\mathrm{V}) \\ U & \text { mean flow velocity }(\mathrm{m} / \mathrm{s}) \\ v & \text { acoustic velocity }(\mathrm{m} / \mathrm{s}) \\ x & \text { spatial coordinate }(\mathrm{m}) \\ \boldsymbol{x} & \text { state vector }(-) \\ Z & \text { specific impedance }(-) \\ \alpha & \text { area ratio }(-) \\ \zeta & \text { loss coefficient }(-) \\ \rho & \text { density }\left(\mathrm{kg} / \mathrm{m}^{3}\right) \\ \sigma & \text { time-delay variance }(\mathrm{s})\end{array}$

$\begin{array}{ll}\tau & \text { mean time-delay }(\mathrm{s}) \\ \omega & \text { circular frequency }(1 / \mathrm{s})\end{array}$

Subscripts and superscripts

c cold

d downstream

eff effective

$G \quad$ loudspeaker

h hot

$R \quad$ reflection coefficient

$\mathrm{T}$ matrix conjugate transpose

u upstream

$-1 \quad$ matrix inverse

$+\quad$ pseudo-inverse

. derivative with respect to time 